\title{
Molecular authentication of the traditional Chinese medicine Tongren Dahuoluo Wan and its alternative formulation
}

\author{
Jikun WANG ${ }^{1}$, Jing DU ${ }^{2}$, Meng CAO ${ }^{1}$, Lu YAO ${ }^{2}$, Suhua XIE ${ }^{2}$, Jiafu CHEN ${ }^{2}$, Xingbo ZHAO(ه) ${ }^{1}$ \\ 1 National Engineering Laboratory for Animal Breeding; Key Laboratory of Animal Genetics, Breeding and Reproduction, Ministry of \\ Agriculture; College of Animal Science and Technology, China Agricultural University, Beijing 100193, China \\ 2 Research Institute of Beijing Tongrentang Co., Ltd., Beijing 100079, China
}

\begin{abstract}
Tongren Dahuoluo Wan has been a popular traditional Chinese medicine in international pharmaceutical markets for hundreds of years. Leopard bone powder is the key element in its formulation. However, the leopard has been listed for wildlife conservation, which limits the use of the leopard bone supplies. Therefore, an alternative formulation which substitutes leopard bone with zokor bone in the formula of Tongren Dahuoluo Wan is now manufactured. To develop a simple and reliable molecular method for authenticating the two patent medicines, mitochondrial nucleotide polymorphic sites of $12 \mathrm{~S} r R N A$, $C O I$ and $C y t b$ genes were screened in leopard and zokor bones, and nine pairs of species-specific primers were verified for discriminating the two species. For the patent medicine authentication, we set up a molecular diagnostic assay to resolve the difficulties of low concentration of target DNAs and presence of PCR-inhibitory substances in this complex medicine, and successfully confirmed leopard or zokor content using the nine pairs of species-specific primers. We recommend a common technical strategy for authentication of species origins in traditional Chinese medicine, and discuss the experimental solutions for technical problems of molecular diagnostic assays.
\end{abstract}

Keywords Tongren Dahuoluo Wan, molecular diagnostic assay, Eospalax baileyi, Panthera pardus, species-specific primers

\section{Introduction}

Tongren Dahuoluo Wan is a traditional Chinese medicines (TCMs) produced by Beijing Tongrentang which is a famous time-honored brand in the TCM industry. Leopard

Received February 10, 2017; accepted April 10, 2017

Correspondence: zhxb@cau.edu.cn
(Panthera pardus) bone has been regarded as a key element of Tongren Dahuoluo Wan. Due to its specific use for treating wind-cold-dampness arthralgia-syndrome, Tongren Dahuoluo Wan has been extensively produced and widely used. However, leopard has been listed in Appendix I of CITES (Convention on International Trade in Endangered Species) and the Red List of IUCN (International Union for the Conservation of Nature), which lists species that are the most endangered and threatened with extinction. With an increasing international demand for Tongren Dahuoluo Wan, compliance with legality, and hence the CITES status, Beijing Tongrentang developed an alternative Tongren Dahuoluo Wan, which replaced the bone of leopard with zokor (Eospalax baileyi), and previous studies reported the rational substitute of the bone content using the zokor for the leopard, based on medical bioactivities which included anti-inflammatory effect, dissipation of blood stasis effect and antioxidant capacity ${ }^{[1,2]}$. Therefore, it is of great importance to be able to authenticate Tongren Dahuoluo Wan with this alternative formulation.

Even though morphometrics is reliable for distinguishing leopard bones from zokor bones, it is not applicable to identifying the sources of ingredients in processed medicine. Over recent decades, chemo-profiling techniques, such as high performance liquid chromatography ${ }^{[3]}$, gas chromatography ${ }^{[4]}$ and near-infrared spectroscopy ${ }^{[5]}$ have been widely applied to medical ingredient identification. By addressing the detection of certain compounds, these methods have complemented the limitations of morphological identification, yet cannot reliably provide species authentication. Therefore, molecular diagnostic assays have been increasingly utilized for authenticating particular species signatures in $\mathrm{TCMs}^{[6,7]}$.

Since highly processed patent medicines usually contains poor nuclear DNAs, the high copy number of mitochondrial DNA (mtDNA) is a suitable target for 
species testing ${ }^{[8]}$. Therefore, we set up a testing system for discrimination of leopard and zokor contents. Our testing system includes a series of species-specific primers designed for mtDNA $12 S$ rRNA, COI and Cytb genes, and an internal positive control as indicator to monitor the PCR-inhibitory substances in Tongren Dahuoluo Wan formulations.

\section{Materials and methods}

\subsection{Materials}

Leopard (P. pardus) bones, zokor (E. baileyi) bones and Tongren Dahuoluo Wan pills were provided by the Research Institute of Beijing Tongrentang Co., Ltd.

Traditionally, Tongren Dahuoluo Wan consists of 50 ingredients; detail information is listed in Table S1.

\subsection{DNA extraction from bones and patent pills}

Briefly, bones were ground into powder and incubated overnight at $55^{\circ} \mathrm{C}$ with lysis buffer $\left(10 \mathrm{mmol} \cdot \mathrm{L}^{-1}\right.$ Tris- $\mathrm{HCl}$ $\mathrm{pH} 8.0,200 \mu \mathrm{g} \cdot \mathrm{mL}^{-1}$ Proteinase $\mathrm{K}, 0.1 \mathrm{~mol} \cdot \mathrm{L}^{-1}$ EDTA pH $8.0,0.5 \%$ SDS), followed by centrifugation for $15 \mathrm{~min}$ at $12000 \mathrm{~g}$ and taking the supernatant for phenol-chloroform extraction ${ }^{[9]}$. DNA was detected by $0.7 \%$ agarose gel electrophoresis. DNA extraction from Tongren Dahuoluo Wan formulations was based on the protocol of Cao et al. ${ }^{[6]}$.

\subsection{Primers}

Nine pairs of species-specific primers were designed according to mitochondrial genome sequences of leopard (NC 010641) and zokor (JN540033). Primers specific for leopard and zokor were designed based on their SNP sites detected in $12 S$ rRNA, COI and Cytb gene regions, including five pairs of species-specific primers for leopards and four pairs of species-specific primers for zokors. PCR primer information was listed in Table S2.

\subsection{PCR amplification and sequencing}

The PCR reaction mixture $(25 \mu \mathrm{L})$ included $2.5 \mu \mathrm{L}$ of $10 \times$ Taq PCR buffer, $0.5 \mu \mathrm{L}$ of Taq DNA polymerase, $0.5 \mu \mathrm{L}$ primers $\left(10 \mu \mathrm{mol} \cdot \mathrm{L}^{-1}\right), 0.5 \mu \mathrm{L}$ of dNTPs $\left(10 \mathrm{mmol} \cdot \mathrm{L}^{-1}\right)$, $20 \mu \mathrm{L}$ of distilled water and $1 \mu \mathrm{L}$ of DNA template. In addition, $10 \mathrm{ng}$ PCR product of zokor DNA (preserved in our laboratory) were added to the PCR mixture and used as the internal positive control. The reaction was performed under the following conditions: $95^{\circ} \mathrm{C} 5 \mathrm{~min}$, and 35 cycles of $95^{\circ} \mathrm{C} 30 \mathrm{~s}, 55-70^{\circ} \mathrm{C} 30 \mathrm{~s}$ and $72^{\circ} \mathrm{C} 30 \mathrm{~s}$, at last $72^{\circ} \mathrm{C}$ $5 \mathrm{~min}$. The products were then analyzed by $1.5 \%$ agarose gel electrophoresis.

\subsection{Phylogenetic cluster analysis}

The COI sequences of zokor and leopard were used as anchors to blast homologous sequences in GenBank. The targeted sequences were defined as species E. baileyi or $P$. pardus, and potential incorrect sequences were excluded manually. The extracted data sets are listed in Table S3. Consensus NJ (Neighbor-Joining) trees were constructed by 1000 bootstraps and depicted using MEGA6 software $^{[10]}$.

\section{Results}

\subsection{DNA extraction from animal bones and pills}

Total DNA was extracted from animal bones in large amounts but was degraded, and from the Tongren Dahuoluo Wan pills, the DNA extracted was badly degraded (Fig. 1).

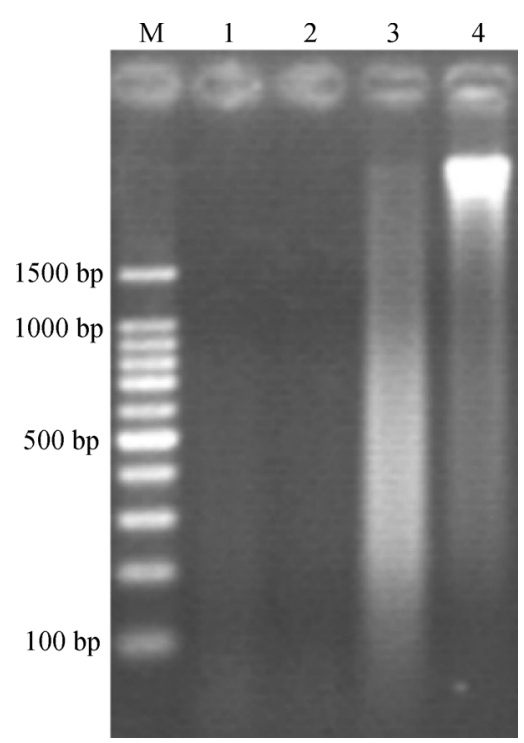

Fig. 1 Gel electrophoresis of DNA extracted from Tongren Dahuoluo Wan pills and animal bones. M, 100 bp DNA Ladder marker; 1, Tongren Dahuoluo Wan (original); 2, Tongren Dahuoluo Wan (alternative); 3, zokor bone; 4, leopard bone.

3.2 PCR identification of patent pills using species-specific primers

The internal positive control generated target fragments, indicating no PCR inhibitory substances were present in bone DNA extracts. All species-specific primers generated correct amplicons, showing evidence of accurate authentication of the medicine (Fig. 2). 


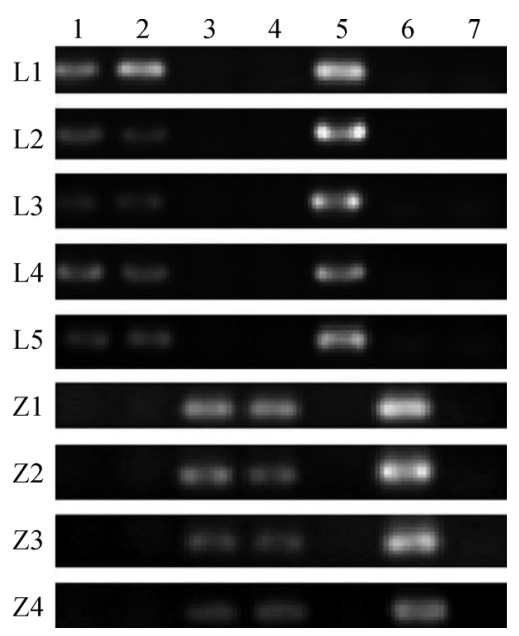

Fig. 2 PCR identification of patent medicines of Tongren Dahuoluo Wan original and alternative pills using species-specific mtDNA primers. 1, Tongren Dahuoluo Wan (original with leopard); 2, internal positive control (leopard); 3, Tongren Dahuoluo Wan (alternative with zokor); 4 , internal positive control (zokor); 5, leopard bone; 6, zokor bone; 7, blank control. L1-L5, specific primers for leopard; Z1-Z4, specific primers for zokor.

\subsection{Sequence verification}

For the accuracy of the authentication, all PCR fragments were subsequently sequenced. For the $12 S r R N A$ fragment, the zokor or leopard medicines gave the corresponding complete identical sequence (NC_010641 for leopard, and JN540033 for zokor), while the leopard medicine had one polymorphic site in the $C O I$ fragment and 10 sites in the $C y t b$ fragments, and the zokor medicine had one

(a)

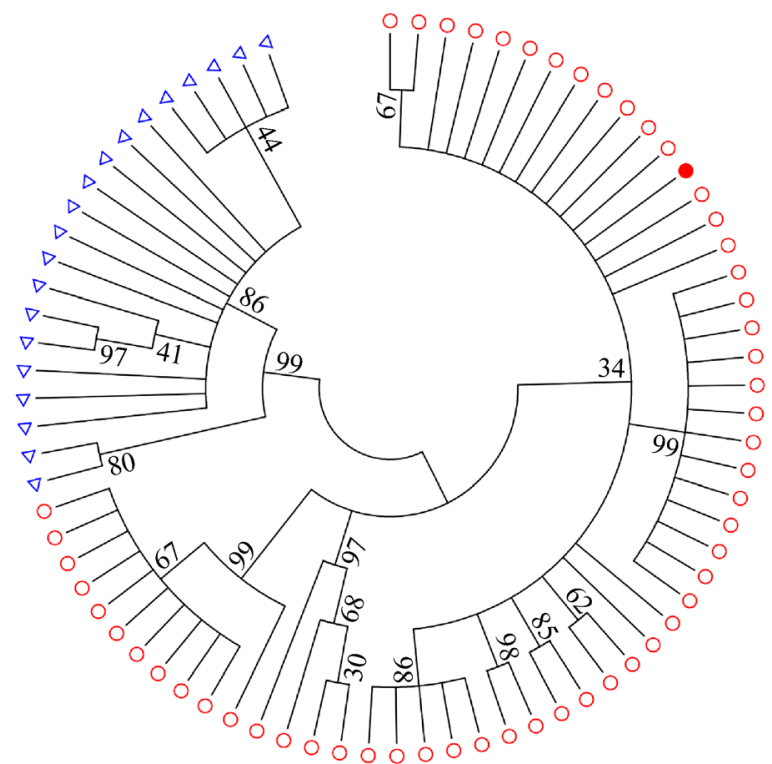

polymorphic site in the $C O I$ fragment and 10 sites in the Cytb fragments (Table S4; Table S5). A Phylogenetic tree for zokor or leopard medicine respectively was constructed using COI sequences which were joined to form an integrated sequence. The two medicines were separately clustered with GenBank sequences, including 56 alignments of E. baileyi and 20 of $P$. pardus (Table S3). Both phylogenetic consensus trees resulted in two apparent clusters, which represented E. baileyi and P. pardus, respectively (Fig. 3). The sequences generated by zokor specific primers was found in the E. baileyi clade (Fig. 3a), while the sequences generated by leopard specific primers were found in the P. pardus clade (Fig. 3b). The results clearly verified the validity and fidelity of the medicine identification.

\section{Discussion}

For CITES member states to enforce legislation and to prosecute cases of illegal trade, a reliable method of species identification is needed ${ }^{[11]}$. TCM preparations are of different types, including pills, capsules, powders and tablets $^{[12]}$, and their chemical composition is quite complex, which makes it difficult to identify animal origins by traditional taxonomic and chromatographic methods ${ }^{[13]}$. Molecular authentication of particular species signatures in TCMs using DNA markers can meet the requirements for correctness and accuracy.

Molecular authentication of TCM suffers from a series of technical problems, mainly arising from DNA extraction, PCR assay and impacts the resulting authenticity, as illustrated in Fig. 4. DNAs preserved in TCMs are actually

Fig. 3 Phylogenetic clustering of the leopard and zokor medicines using the COI sequences. Red circles represent the Eospalax baileyi individuals, the blue triangles represented those from Panthera pardus, and the solid node represents the medicine sequences. The numbers at each node represent the posterior probability support values.

(b)

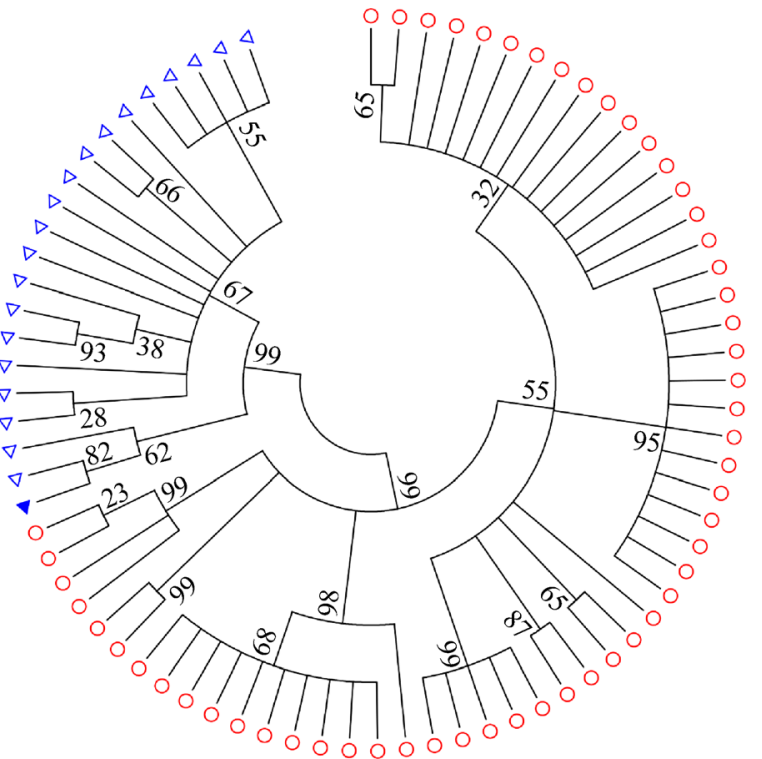




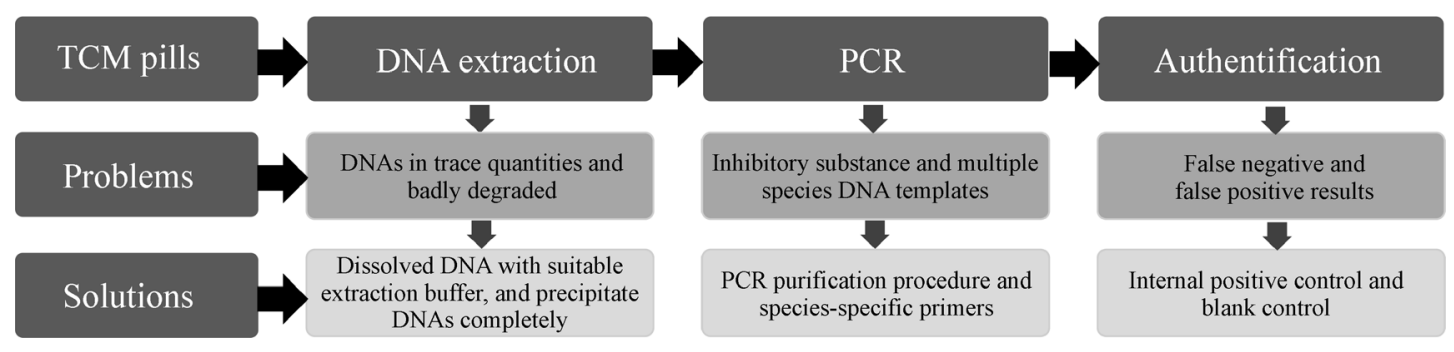

Fig. 4 Strategy for molecular authentication of animal products used in Chinese traditional medicines

complex and in various states of degradation, therefore it is crucial to preserve the available quantity as much as possible for further enzymatic manipulation. The extraction method needs to avoid overly aggressive treatments, such as high temperatures or use of strong detergents ${ }^{[14]}$. So far, the phenol-chloroform method has proven to be a simple and effective technique for extracting DNA from complex samples ${ }^{[6,9]}$. In addition, concentration of DNA using membranes ${ }^{[15]}$ or binding DNA to silica ${ }^{[16]}$ can be an effective but expensive alternative for TCM DNA extraction. The sensitivity and kinetics of PCR may be dramatically reduced by PCR-inhibitory substances (e.g., plant polyphenol) ${ }^{[17]}$ in TCMs, so purification of TCM DNAs using QIAquick ${ }^{\mathrm{TM}}$ Purification Kit (Qiagen, Hilden, Germany) and setting the internal positive control to monitor the presence or absence of the PCR-inhibitory factors $^{[12,13]}$ is necessary. We also used a blank control to monitor false positive results. When both the sample and the internal positive control are negative, the TCM DNA contains PCR-inhibitory substances and the DNA extract needs to be purified.

In recent years, various molecular markers have been developed for authentication of TCMs, including random amplified polymorphic DNA (RAPD), inter-simple sequence repeats (ISSR) and restriction fragment length polymorphism (RFLP) ${ }^{[18-21]}$. In addition to poor reproducibility, RAPD, ISSR and RFLP can be easily affected by DNA degradation, thus they are not suitable for identification of highly processed samples. Simple sequence repeats ${ }^{[22]}$ have also been used in authenticating medicinal materials, but the products amplified from each allele are usually short fragments, subsequently complicating reliability scores by standard agarose gel electrophoresis ${ }^{[23]}$. Next-generation sequencing technology is a powerful tool in identifying biological ingredients in $\mathrm{TCMs}^{[13,24]}$. The advantage of quality evaluation of TCM preparations based on metagenomic approach via high-throughput sequencing is that it not only detects the prescribed species but also any contaminating species. However, it is also restricted by DNA degradation during TCM processing or the lack of reference sequence in the database.

Species-specific primers to authenticate target species (e.g., E. baileyi) in TCMs are simple and rapid, with low thresholds and high efficiency, and the increasing availability of mitogenome sequences deposited in EMBL or GenBank will enable a wide range of species-specific primers to be designed.

\section{Conclusions}

In this study, a range of mtDNA SNPs were detected between $E$. baileyi and P. pardus. With the specific primers designed from SNP sites and an internal positive control as an indicator to monitor PCR-inhibitory substances, the animal origin of contents of Tongren Dahuoluo Wan formulations were precisely identified. In conclusion, molecular diagnostic assays are considered invaluable tools for authenticating particular species signatures of TCMs.

Supplementary materials The online version of this article at http://dx. doi.org/10.15302/J-FASE-2017157 contains supplementary materials (Tables S1-S5).

Acknowledgements The research was supported by projects of Beijing Municipal Science \& Technology Commission (D08080203640903) and National Natural Science Foundation of China (31672379). We thank Dr. Hai Xiang (Institute of Genetics and Developmental Biology, CAS) and Dr. Langqing Liu (Wageningen University) for assistance with phylogenetic cluster analysis.

Compliance with ethics guidelines Jikun Wang, Jing Du, Meng Cao, Lu Yao, Suhua Xie, Jiafu Chen, and Xingbo Zhao declare that they have no conflict of interest or financial conflicts to disclose.

This article does not contain any studies with human or animal subjects performed by any of the authors.

\section{References}

1. Ping H. Experimental study on anti-inflammatory effect of Sailong Bone. Liaoning Journal of Traditional Chinese Medicine, 2000, 27 (11): 524-526 (in Chinese)

2. Zhai J Y, Chen X, Jin R, Yue F, Liu D, Li J S. Effect of Zaizao Wan (Sailong bone replacement of leopard bone) against cerebral ischemia. Chinese Journal of Experimental Traditional Medical Formulae, 2016, 22(6): 124-129 (in Chinese)

3. Lee D G, Kang H W, Park C G, Ahn Y S, Shin Y. Isolation and identification of phytochemicals and biological activities of 
Hericium ernaceus and their contents in Hericium strains using HPLC/UV analysis. Journal of Ethnopharmacology, 2016, 184: 219-225

4. Shellie R A, Marriott P J, Huie C W. Comprehensive twodimensional gas chromatography $(\mathrm{GC} \times \mathrm{GC})$ and $\mathrm{GC} \times \mathrm{GC}$-quadrupole MS analysis of Asian and American ginseng. Journal of Separation Science, 2003, 26(12-13): 1185-1192

5. Puchert T, Lochmann D, Menezes J C, Reich G. Near-infrared chemical imaging (NIR-CI) for counterfeit drug identification-a four-stage concept with a novel approach of data processing (Linear Image Signature). Journal of Pharmaceutical and Biomedical Analysis, 2010, 51(1): 138-145

6. Cao M, Wang J, Yao L, Xie S, Du J, Zhao X. Authentication of animal signatures in traditional Chinese medicine of Lingyang Qingfei Wan using routine molecular diagnostic assays. Molecular Biology Reports, 2014, 41(4): 2485-2491

7. Li M, Au K Y, Lam H, Cheng L, Jiang R W, But P P H, Shaw P C. Identification of Baiying (Herba Solani Lyrati) commodity and its toxic substitute Xungufeng (Herba Aristolochiae Mollissimae) using DNA barcoding and chemical profiling techniques. Food Chemistry, 2012, 135(3): 1653-1658

8. Xiang H, Gao J, Yu B, Zhou H, Cai D, Zhang Y, Chen X, Wang X, Hofreiter M, Zhao X. Early Holocene chicken domestication in northern China. Proceedings of the National Academy of Sciences of the United States of America, 2014, 111(49): 17564-17569

9. Kalmár T, Bachrati C Z, Marcsik A, Raskó I. A simple and efficient method for PCR amplifiable DNA extraction from ancient bones. Nucleic Acids Research, 2000, 28(12): E67

10. Tamura K, Stecher G, Peterson D, Filipski A, Kumar S. MEGA6: molecular evolutionary genetics analysis version 6.0. Molecular Biology and Evolution, 2013, 30(12): 2725-2729

11. Linacre A. The use of DNA from non-human sources. Forensic Science International: Genetics Supplement Series, 2008, 1(1): 605-606

12. Whiting M, Williams V, Hibbitts T. In: Alves R R N, Rosa I L, eds. Animals in traditional folk medicine. Springer, 2013, 421-473

13. Coghlan M L, Haile J, Houston J, Murray D C, White N E, Moolhuijzen P, Bellgard M I, Bunce M. Deep sequencing of plant and animal DNA contained within traditional Chinese medicines reveals legality issues and health safety concerns. PLoS Genetics, 2012, 8(4): e1002657

14. Rohland N, Hofreiter M. Comparison and optimization of ancient DNA extraction. BioTechniques, 2007, 42(3): 343-352

15. Leonard J A, Wayne R K, Cooper A. Population genetics of ice age brown bears. Proceedings of the National Academy of Sciences of the United States of America, 2000, 97(4): 1651-1654

16. Hofreiter M, Rabeder G, Jaenicke-Desprs V, Withalm G, Nagel D, Paunovic M, Jambrěsić G, Pääbo S. Evidence for reproductive isolation between cave bear populations. Current Biology, 2004, 14 (1): 40-43

17. Rådström P, Knutsson R, Wolffs P, Lövenklev M, Löfström C. PrePCR processing: strategies to generate PCR-compatible samples. Molecular Biotechnology, 2004, 26(2): 133-146

18. Cheng K T, Tsay H S, Chen C F, Chou T W. Determination of the components in a Chinese prescription, yu-ping-feng san, by RAPD analysis. Planta Medica, 1998, 64(6): 563-565

19. Chang S, Kalok W, But P, Su W W, Pangchui S. Molecular authentication of the Chinese herb Huajuhong and related medicinal material by DNA sequencing and ISSR markers. Journal of Food and Drug Analysis, 2010, 18(18): 161-170

20. Reunova G D, Kats I L, Muzarok T I, Zhuravlev IuN. Polymorphism of RAPD, ISSR and AFLP markers of the Panax ginseng C. A. Meyer (Araliaceae) genome. Russian Journal of Genetics, 2010, 46 (8): 1057-1066

21. Lin T C, Yeh M S, Cheng Y M, Lin L C, Sung J M. Using ITS2 PCR-RFLP to generate molecular markers for authentication of Sophora flavescens Ait. Journal of the Science of Food and Agriculture, 2012, 92(4): 892-898

22. Kim J, Jo B H, Lee K L, Yoon E S, Ryu G H, Chung K W. Identification of new microsatellite markers in Panax ginseng. Molecules and Cells, 2007, 24(1): 60-68

23. Hayashi K, Hashimoto N, Daigen M, Ashikawa I. Development of PCR-based SNP markers for rice blast resistance genes at the Piz locus. Theoretical and Applied Genetics, 2004, 108(7): 1212-1220

24. Cheng X, Su X, Chen X, Zhao H, Bo C, Xu J, Bai H, Ning K. Biological ingredient analysis of traditional Chinese medicine preparation based on high-throughput sequencing: the story for Liuwei Dihuang Wan. Scientific Reports, 2014, 4(1): 5147 